\title{
Research on the Early Strength Agent Effect on the Fatigue Performance of Cement Stabilized Macadam
}

\author{
Jie Yang ${ }^{1, a^{*}}$ \\ ${ }^{1}$ College of Traffic and Civil Engineering of Shan Dong Jiaotong University, Jinan City 250357, \\ China \\ ayyangjie129@163.com
}

\begin{abstract}
Keywords: Early Strength Agent, Cement Stabilized Macadam, Stress Level, Fatigue Life, Weibull Distribution, Fatigue Equation

Abstract. Strength of cement stabilized macadam which added early strength agent improves quickly. This shortens the construction period of road maintenance. This study explores the difference of fatigue performance between the cement stabilized macadam which added early strength agent and the ordinary cement stabilized macadam. Test fatigue lives under different stress levels. These fatigue lives in line with double-parameter Weibull Distribution. Then determine the two parameters of Weibull Distribution. On this basis, establish the fatigue equations of each early strength agent amount and determine the regression coefficients. The results show that early strength agent can improve fatigue life of cement stabilized macadam, but it makes cement stabilized macadam become more sensitive to stress level.
\end{abstract}

\section{Introduction}

It has been shown [1], the early strength cement stabilized macadam strength forming needs very short time, this shorten road maintenance schedule to provide convenient conditions. Since cement stabilized macadam is quasi-brittle material [2,3], when load repetition over a certain number of times, cement stabilized macadam base tensile strain reaches the limit, then cracking, that is fatigue cracks . In other words, the fatigue properties of cement stabilized macadam directly affect their life. The present study is based on previous research [1], analysis the fatigue performance differences between cement stabilized macadam which added early strength agent and the ordinary cement stabilized macadam, with using MTS testing machine.

\section{Raw Materials}

Cement. $32.5 \mathrm{R} \mathrm{P} \cdot \mathrm{S}$ Cement. The main technical nature is in line with requirements.

Aggregate. Limestone. The mineral aggregate crushing value measurement is less than $30 \%$. Continuous gradation is in line with regulatory requirements. The maximum nominal diameter is less than $26.5 \mathrm{~mm}$.

Early Strength Agent. Early strength agent is CS-I type ultra-early strength material which used in cement stabilized base. It was invented by Shuanfa Chen who work at Chang'an University. The early strength agent is pale yellow powder. The main mineral component is calcium silicate, calcium aluminates, calcium sulphoaluminate, sucrose, etc.

\section{Main Test}

Specimens Molding. Accordance with the test procedures [4], determine the maximum dry density and optimum water content by the compaction test method. By mixture design without the early strength agent, the best cement dose is $5 \%$. In the fatigue properties test, the early strength agents were taken $0 \%, 4 \%, 8 \%$ and $12 \%(Z 0, Z 4, Z 8$ and $Z 12)$. The early strength agent should be mixed well with cement before mixing with aggregate. This test uses beam specimens which size is $10 \mathrm{~cm} \times 10 \mathrm{~cm} \times$ $40 \mathrm{~cm}$. The compaction is $98 \%$ [5]. Specimens forming process accord to the relevant test procedures [4], the standard regimen. 


\section{Fatigue Test.}

Test methods and test parameters. Fatigue test uses control stress mode, the stressing schema is sinusoidal curve. Test error is less than $20 \%$. The temperature is between $10 \sim 20^{\circ} \mathrm{C}$. Loading error is less than $2 \%$. Test was carried out on MTS testing machine, the regulator, under load, to be stable after the indicated value by applying an alternating load. The characteristics of the load cycle fatigue testing, the value $R$ is 0.02 . Loads at three dividing point. The span is $30 \mathrm{~cm}$. Load frequency is $10 \mathrm{~Hz}$.

Data Processing. Test cement stabilized macadam fatigue life $N$ in each early strength agent amount when stress levels $S$ are $0.7,0.75,0.8$ and 0.85 . Since fatigue life of cement stabilized macadam is a random quantity, its discrete greater need to study the probability distribution to more accurately and comprehensively establish fatigue equation. Some studies have shown the equivalent fatigue life $\bar{N}$ of ordinary concrete is in line with double-parameter Weibull Distribution. That is Eq. 1.

$$
\ln \ln \frac{1}{1-\rho}=m \ln \bar{N}-\ln t_{0}
$$

Where, $m$ is shape parameter, $t_{0}$ is scale parameter, $\rho$ is the probability of failure, $\bar{N}=N^{1-R}$.

By the fatigue life of cement stabilized macadam which added each early strength agent amount and failure probability $\rho$ in each stress level $S$, we get Fig. 1 to Fig. 4.

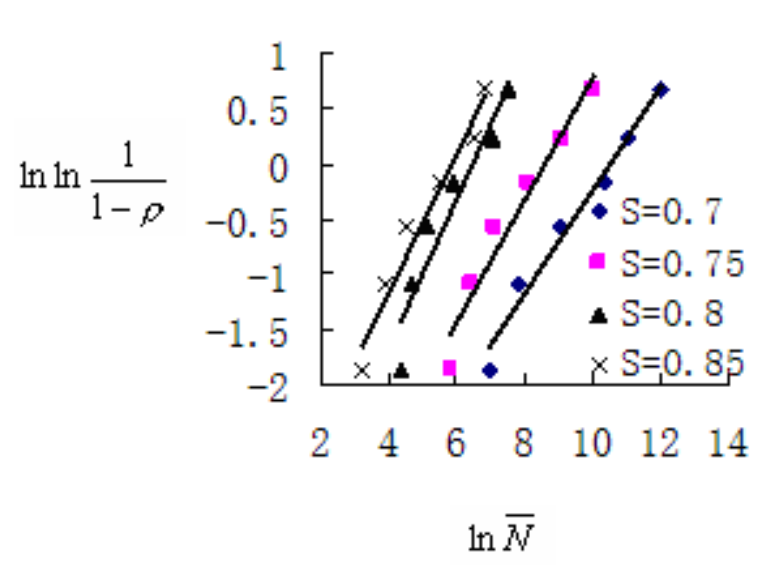

Fig. 1. Verification $Z 0$ is in line with Weibull Distribution

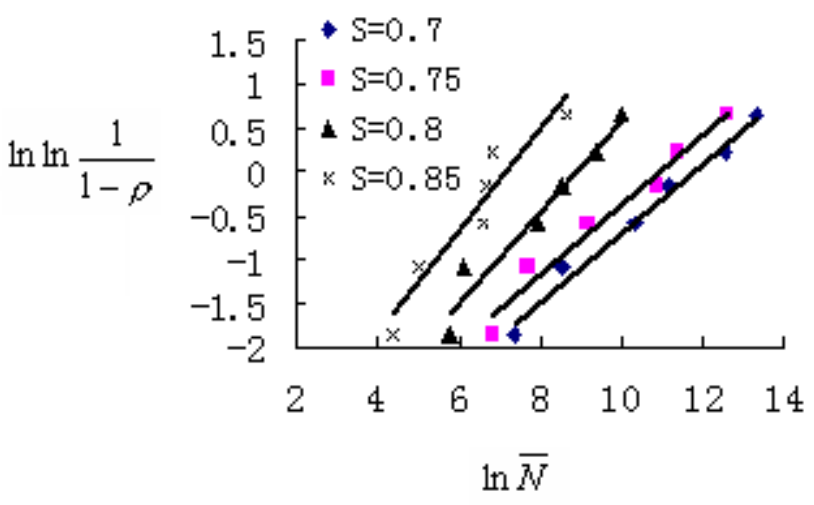

Fig. 3. Verification $Z 8$ is in line with Weibull distribution

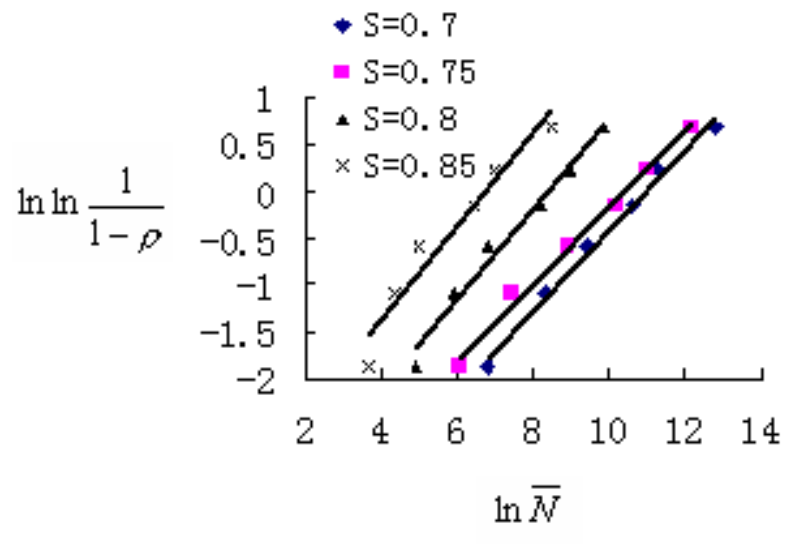

Fig. 2. Verification $Z 4$ is in line with Weibull distribution

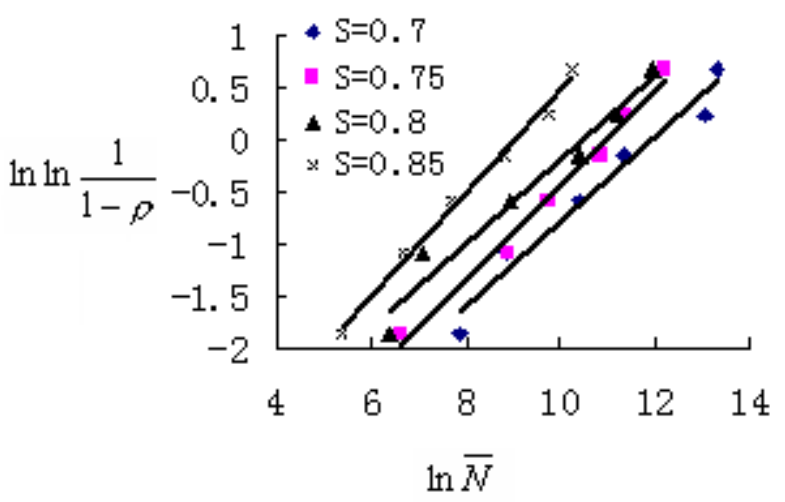

Fig. 4. Verification $Z 12$ is in line with Weibull distribution

As can be seen from Fig.1 to Fig. $4, \ln \ln \frac{1}{1-\rho}$ and $\ln \bar{N}$ has a good linear relationship. Then determine each parameter $m$ and $l n t_{0}$ under each stress level $S$, as shown in Table 1 . Therefore, we can use double-parameter Weibull Distribution deal with the resulting test data.

Establishment of fatigue equation. After analysis the fatigue test of cement stabilized macadam results is the fatigue equation. That is Eq.2.

$\lg S=\lg a-b(1-R) \lg N$ 
Where, $a, b$ are undetermined coefficients of fatigue test, the remaining symbols with the same meaning.

Regression coefficients and correlation coefficients in Weibull distribution

Table 1

\begin{tabular}{ccccc}
\hline Type & $S$ & $m$ & $\operatorname{lnt} t_{0}$ & $R$ \\
\hline \multirow{3}{*}{$Z 0$} & 0.70 & 0.4675 & 4.9288 & 0.9875 \\
& 0.75 & 0.5536 & 4.7757 & 0.9739 \\
& 0.80 & 0.6800 & 4.3996 & 0.9494 \\
$Z 4$ & 0.85 & 0.6182 & 3.6185 & 0.9806 \\
\hline \multirow{2}{*}{$Z 8$} & 0.70 & 0.4249 & 4.6734 & 0.9951 \\
& 0.75 & 0.4002 & 4.1910 & 0.9962 \\
& 0.80 & 0.4820 & 4.0709 & 0.9885 \\
& 0.85 & 0.4948 & 3.3601 & 0.9689 \\
\hline \multirow{2}{*}{$Z 12$} & 0.70 & 0.3978 & 4.6600 & 0.9916 \\
& 0.75 & 0.4047 & 4.4150 & 0.9837 \\
& 0.80 & 0.5257 & 4.6580 & 0.9763 \\
& 0.85 & 0.5882 & 4.2066 & 0.9532 \\
\hline
\end{tabular}

Determine the undetermined coefficients $a$ and $b$ value as follows: By substitution of the regression parameters $m$ and $l n t_{0}$ into Eq.1, can be calculated equivalent fatigue life $\bar{N}$ for each stress level $S$ corresponding to different failure probability $\rho$. According to $S$ and $\bar{N}$, the undetermined coefficients $a, b$ and the correlation coefficient can be determined, as shown in Table 2 .

The fatigue equation regression coefficients and correlation coefficient

Table 2

\begin{tabular}{ccccc}
\hline Type & $\rho$ & $a$ & $b$ & $R$ \\
\hline \multirow{3}{*}{$Z 0$} & 0.05 & 0.901 & 0.060 & 0.9979 \\
& 0.10 & 0.955 & 0.054 & 0.9982 \\
$Z 4$ & 0.50 & 1.035 & 0.040 & 0.9862 \\
\hline \multirow{2}{*}{$Z 4$} & 0.05 & 0.908 & 0.061 & 0.9860 \\
& 0.10 & 0.972 & 0.055 & 0.9869 \\
& 0.50 & 1.115 & 0.044 & 0.9756 \\
\hline \multirow{2}{*}{$Z 12$} & 0.05 & 1.041 & 0.088 & 0.9741 \\
& 0.10 & 1.086 & 0.070 & 0.9855 \\
& 0.50 & 1.132 & 0.043 & 0.9840 \\
\hline
\end{tabular}

The regression coefficient $a$ value in Table 2 reflects the anti-fatigue properties of the material; greater $a$ value indicates better fatigue properties of materials. As can be seen from the test results, the probability of failure in the same conditions, $Z 0$ is the minimum and $Z 12$ is the maximum. The $a$ value increases by more early strength agent amount. To the failure probability $5 \%$ as an example, the $a$ value of cement stabilized macadam added early strength agent is generally higher than ordinary cement stabilized macadam, $Z 12$ and $Z 8$ increase respectively $50 \%$ and $9.4 \%$, compared with $Z 0$. This shows that the early strength agent improve fatigue performance of cement stabilized macadam.

The regression coefficient $b$ value in Table 2 represents sensitivity of fatigue life with stress. Early strength cement stabilized macadam was also significantly higher than ordinary cement stabilized macadam. To the failure probability $5 \%$ as an example, the $b$ value $Z 12$ and $Z 8$ respectively increased by $51.7 \%$ and $46.7 \%$, compared with $Z 0$. This indicates fatigue curves of early strength cement stabilized macadam steepness, sensitivity of fatigue life with stress levels also increased. 


\section{Conclusions}

(1)Fatigue life of cement stabilized macadam is in line with the double-parameters Weibull Distribution; we can use it to establish fatigue equation;

(2)Early strength agent can significantly improve the cement stabilized macadam fatigue life, improve the fatigue performance;

(3)By added early strength agent, fatigue life of cement stabilized macadam become more sensitive to stress level.

\section{Acknowledgement}

This work was financially supported by the Fund Project of Shan Dong Jiaotong University (Z201322) "Research on Fatigue Performance of Early Strength Cement Stabilized Macadam Based on MTS Tests".

\section{References}

[1] Jie Yang, Feng-bin Sun, Zu-zhong Li. Shanxi Architecture, Vol. 3(5)( 2005) 174-175.(In Chinese) [2] Jian-xiao Tian, Shuan-fa Chen, Bo Peng. Journal of Chang'an University(Natural Science Edition), Vol. (5)(2003)24-27. (In Chinese)

[3] Hong-chang Wang, Xiao-ming Huang, Zhi Fu. Journal of Traffic and Transportation Engineering, Vol. 5(2) (2005)38-41.(In Chinese)

[4] JTG E51-2009, Test Methods of Materials Stabilized with Inorganic Binders for Engineering, Beijing, 2009. (In Chinese)

[5]JTJ 034-2000, Technical Specifications for Construction of Highway Road base, Beijing, 2000.(In Chinese) 